\title{
Manipulation of the physiology of clavulanic acid production in Streptomyces clavuligerus
}

\author{
Paul R. Ives $\nmid$ and Michael E. Bushell \\ Author for correspondence: Michael E. Bushell. Tel: +44 1483 259277. Fax: +44 1483300374. \\ e-mail:m.bushell@surrey.ac.uk
}

Microbial Physiology and Ecology Group, University of Surrey, Guildford, Surrey GU2 5XH, UK

\begin{abstract}
This paper reports a novel use of cluster analysis for the identification of intermediary metabolites that are produced at rates closely correlated with those of antibiotic biosynthesis. This information was used to devise culture feeds resulting in enhanced production of clavulanic acid, an antibiotic of current worldwide commercial interest. The feeding strategies apparently alleviated a rate-limiting supply of the $C_{3}$ precursor of clavulanic acid. $C_{3}$ limitation may be a consequence of unusual nitrogen and carbon metabolism in Streptomyces clavuligerus. This approach has potential as a generic method for influencing biosynthetic pathway fluxes using feeds without knowledge of the biosynthetic pathway.
\end{abstract}

Keywords: secondary metabolism, clavulanic acid, nutrient feeds, cluster analysis

\section{INTRODUCTION}

Bioreactor feeding strategies have been used as a means of (I) controlling growth rate, (II) prolonging stationary phase and (III) supplying feeds of potentially deleterious precursors to the culture at subinhibitory concentrations. Strategy III is well established in antibiotic production processes (Queener \& Swartz, 1979) and we have applied strategy II to antibiotic and monoclonal antibody production in, respectively, Streptomyces (Bushell, 1988) and hybridoma (Bushell et al., 1994) cell cultures. The control of growth rate (strategy I) has been used to avoid oxygen limitation during yeast production (Reed \& Peppler, 1973).

Clavulanic acid is a potent inhibitor of $\beta$-lactamases of both Gram-positive and Gram-negative pathogenic bacteria (Reading \& Cole, 1977). It exerts a marked synergistic effect with $\beta$-lactamase-susceptible penicillins against many penicillin-resistant infections (Brown, 1986). Augmentin, a commercial combination of amoxycillin and clavulanic acid, is currently prescribed in 117 countries, when antibiotic-resistant infections are suspected. Clavulanic acid will shortly attain generic status and is, therefore, of widespread interest.

Streptomyces clavuligerus was isolated from a South American soil sample. It produces at least 21 secondary metabolites (Hopwood, 1981), including several $\beta$ -

†Present address: Gist-brocades BV, PO Box 1, 2600 MA Delft, The Netherlands. lactam antibiotics. The sugar utilization pattern is unusual in that glucose is not readily assimilated.

Radiolabelled feeding experiments have indicated that arginine and ornithine are efficiently incorporated into clavulanic acid (Townsend \& Ho, 1985a ; Romero et al., 1986). Despite evidence (Romero et al., 1986) that arginase (converting arginine to ornithine) is active in cultures of $S$. clavuligerus, ornithine is not a direct precursor. A study in which radiolabelled amino acids were fed to arginine auxotrophs demonstrated that ornithine has to be converted to arginine (via a sequence analogous to the mammalian urea cycle) before it can be incorporated into clavulanic acid (Valentine et al., 1993). The remainder of the clavulanic acid molecule is derived from a $\mathrm{C}_{3}$ precursor. Stirling \& Elson (1979) obtained evidence for incorporation of glycerol and a later report suggested that $\mathrm{D}$-glycerate could act as an intermediate between glycerol and clavulanic acid (Townsend \& Ho, 1985b). A mechanism accounting for clavulanic acid and valclavam (a related structure produced by Streptomyces antibioticus) biosynthesis from arginine and glycerol has been presented (Baldwin et al., 1994). However, more recent evidence (Pitlik \& Townsend, 1997) has shown that hydrogen at the C-2 position of glycerate is lost in the biosynthesis of clavulanic acid, suggesting that the $\mathrm{C}_{3}$ precursor could be pyruvate.

We have examined the necessity for growth-rate downregulation for the induction of secondary metabolism (Bushell et al., 1997; Clark et al., 1995; Wilson \& Bushell, 1995). These studies led to the development of cyclic fed batch cultures for enhanced production of 
antibiotics. This report includes the evaluation of twostage chemostat cultures, an alternative strategy for achieving growth-rate down-regulation.

\section{METHODS}

Strains and culture media. Streptomyces clavuligerus NRRL 3585 (ATCC 27064) was used throughout. The chemically defined media contained (maximum concentrations, $\mathrm{g} \mathrm{l}^{-1}$ in reverse-osmosis-purified water): glycerol $60, \mathrm{NH}_{4} \mathrm{Cl} 7 \cdot 0$, $\mathrm{KH}_{2} \mathrm{PO}_{4} 3 \cdot 0, \mathrm{~K}_{2} \mathrm{HPO}_{4} 7 \cdot 0, \mathrm{MgSO}_{4} .7 \mathrm{H}_{2} \mathrm{O} 0 \cdot 25, \mathrm{FeSO}_{4} .7 \mathrm{H}_{2} \mathrm{O}$ $0 \cdot 025, \mathrm{CuCl}_{2} 0 \cdot 00053, \mathrm{CoCl}_{2} 0 \cdot 00055, \mathrm{CaCl}_{2} .2 \mathrm{H}_{2} \mathrm{O} 0 \cdot 0138$, $\mathrm{ZnCl}_{2} 0 \cdot 0104, \mathrm{MnCl}_{2} 0 \cdot 0062, \mathrm{Na}_{2} \mathrm{MoO}_{4} 0 \cdot 0003$; reduced to ( $\mathrm{g}$ $1^{-1}$ ) glycerol 15 (C-limited), $\mathrm{NH}_{4} \mathrm{Cl} 1.5$ (N-limited) and $\mathrm{KH}_{2} \mathrm{PO}_{4} 0 \cdot 1$, without $\mathrm{K}_{2} \mathrm{HPO}_{4}$ (P-limited, also contained MOPS at $21 \mathrm{~g} \mathrm{I}^{-1}$ ) prepared as described previously (Bushell et al., 1997).

Cultures were routinely maintained on SV2 agar (Ives, 1995) and grown in $25 \mathrm{ml}$ cultures in $250 \mathrm{ml}$ magnetically stirred Erlenmeyer flasks at $30^{\circ} \mathrm{C}$. An SV2 broth culture was inoculated with colonies from a plate. The bioreactor inoculum regime was as described previously (Lynch \& Bushell, 1995).

Bioreactor culture. The Braun Biolab bioreactors used had working volumes of $1.5 \mathrm{l}$. Agitation was provided by disc turbine impellers rotating at 750 r.p.m., and sterile air was supplied through a sparger. The temperature was controlled at $30^{\circ} \mathrm{C}$. Cultures were controlled as described previously (Lynch \& Bushell, 1995).

Assays. Biomass (Lynch \& Bushell, 1995), phosphate and ammonia (McDermott et al., 1993) concentrations were determined as described previously. HPLC was used to assay clavulanic acid (Foulstone \& Reading, 1982) and glycerol (Ives, 1995). Experiments showed that clavulanic acid decomposition under the experimental conditions used was insignificant. Other antibiotics produced by this strain were not investigated.

Cell amino acid content was determined using the HPLCbased method of Jones et al. (1981), with the modification of Joseph \& Marsden (1986). Cells were disrupted following suspension in $0.05 \mathrm{M}$ glycine buffer, $\mathrm{pH} 9.5(250 \mathrm{ml} 0.2 \mathrm{M}$ glycine, $96 \mathrm{ml} 0 \cdot 2 \mathrm{M} \mathrm{NaOH}$ and $0 \cdot 1 \mathrm{M} \mathrm{KCI}$ to $1 \mathrm{l}$ ). Single $1 \mathrm{ml}$ volumes were sonicated in $30 \mathrm{~s}$ pulses for $2 \mathrm{~min}$ at $4{ }^{\circ} \mathrm{C}$ at intensity 3 using an Ultrasonic Processor XL sonicator (Heat Systems) fitted with a micro tip. Cell-free extracts were prepared by centrifugation at $12000 \mathrm{~g}$ for $15 \mathrm{~min}$ at $4^{\circ} \mathrm{C}$ and used immediately or frozen at $-20^{\circ} \mathrm{C}$. Derivatization was achieved by adding $200 \mu \mathrm{l}$ OPT-thiol reagent (Sigma) to $50 \mu \mathrm{l}$ samples. After centrifugation at $10000 \mathrm{~g}$ for $10 \mathrm{~min}$, samples $(25 \mu \mathrm{l})$ were injected onto a $5 \mu \mathrm{m}$ Hypersil ODS column (HPLC Technology) of length $150 \mathrm{~mm}$ and internal diameter $4.6 \mathrm{~mm}$ and eluted with two mobile phases. The initial gradient was $0-10 \%$ of mobile phase A $(800 \mathrm{ml} 0.05 \mathrm{M}$ $\mathrm{NaH}_{2} \mathrm{PO}_{4}$ at pH 5.5 and $200 \mathrm{ml}$ methanol) in mobile phase B $(200 \mathrm{ml}$ of the same phosphate buffer solution and $800 \mathrm{ml}$ methanol) applied over $10 \mathrm{~min}$, followed by $10-85 \%$ in $30 \mathrm{~min}, 85-0 \%$ in $5 \mathrm{~min}$ and finally $10 \mathrm{~min}$ equilibration with $A$ at $1 \mathrm{ml} \mathrm{min}-1$. The eluate was monitored at $340 \mathrm{~nm}$. Using this relatively simple single-wavelength detection system we were unable to resolve peaks for glycine, proline or glutamate with sufficient certainty to include concentrations of these amino acids in our calculations. Asparagine concentrations were too small for accurate quantification and were assumed to be insignificant during the subsequent calculations.
Two-stage chemostat culture. The dilution rate was $0.05 \mathrm{~h}^{-1}$ in both vessels; all of the culture flowed from stage 1 to stage 2 without further additions. The rationale behind this experimental design was that the residual concentration of $\mathrm{NH}_{4} \mathrm{Cl}$ in the culture flowing from stage 1 would act as the nitrogen source for the culture in stage 2 . In two-stage chemostat culture, the growth rate in stage 2 is not equal to the dilution rate, and is determined by comparing the increase in biomass concentration in stage 2 with that in stage 1 . The value of the growth rate was calculated using the expression:

$\mu_{2}=D_{2}-D_{1,2} \cdot\left(x_{1} / x_{2}\right)$

(Pirt, 1975) where: $\mu_{2}$ is the specific growth rate in stage $2, D_{2}$ is the dilution rate in stage $2, D_{1,2}$ is the partial dilution rate in stage 2 , accounting for the material transferred from stage 1 (in this case, equal to $D_{2}$ ), $x_{1}$ is the steady-state biomass concentration in stage 1 and $x_{2}$ is the steady-state biomass concentration in stage 2. Estimation of the ammonia concentrations in each stage allowed the calculation of values for substrate affinity and inhibition constants using the equation

$\mu=\mu_{\max }\left[\frac{s}{K_{\mathrm{s}}+s}\right]\left[\frac{K_{\mathrm{i}}}{K_{\mathrm{i}}+I}\right]$

where $\mu$ is the specific growth rate, $\mu_{\max }$ is the maximum specific growth rate, $K_{\mathrm{s}}$ is the substrate affinity constant, $K_{\mathrm{i}}$ is the inhibition constant, $s$ is the steady-state substrate concentration in the culture vessel and $I$ is the inhibitor concentration (in this case, equal to the substrate concentration).

Substituting the values for $s$ from the two stages results in two simultaneous equations allowing solutions for values of $K_{\mathrm{i}}$ and $K_{\mathrm{s}}$.

Cluster analysis. Amino acid production rates $\left(q_{\text {biomass }}^{\text {amino acid }}\right)$ were calculated from the intracellular pool sizes using [amino acid] $\times D$ [ $\mu \mathrm{g}$ (mg biomass) $\left.)^{-1} \mathrm{~h}^{-1}\right]$. A data matrix containing the resultant values and clavulanic acid production rates, obtained over the dilution rate range $0.03-0.09 \mathrm{~h}^{-1}$, was standardized and subjected to cluster analysis using average within-groups linkage with the Unistat statistical analysis package. Results were displayed in the form of a dendrogram.

Reproducibility and replication of experiments. All experimental data were obtained from single cultures. Experiments were carried out in triplicate to ensure that the trends and relationships observed in the culture parameters measured were reproducible. Individual assays were replicated fourfold except for the amino acid assays, which were carried out in duplicate. Experiments were rejected where a chi-squared test indicated significant differences between replicates. Where error bars (which represent standard error) are not shown, they were too small to be visible on the figures presented.

\section{RESULTS AND DISCUSSION}

\section{Effect of growth-rate-limiting nutrient in batch culture}

Bioreactor batch culture using glycerol (C-), $\mathrm{NH}_{4} \mathrm{Cl}(\mathrm{N}-)$ and $\mathrm{KH}_{2} \mathrm{PO}_{4}$ (P-) limited medium formulations resulted in growth-dissociated clavulanic acid production under $\mathrm{N}$ and $\mathrm{P}$ limitation but no production under $C$ limitation (Fig. 1). Exclusive exhaustion of the appropriate nutrients confirmed the identity of the respective growth-limiting substrates (Fig. 1). Specific antibiotic production was threefold higher under $\mathrm{P}$ limitation $\left[0.03 \mathrm{~g}\right.$ clavulanic acid $\left.(\mathrm{g} \text { biomass })^{-1}\right]$ than 


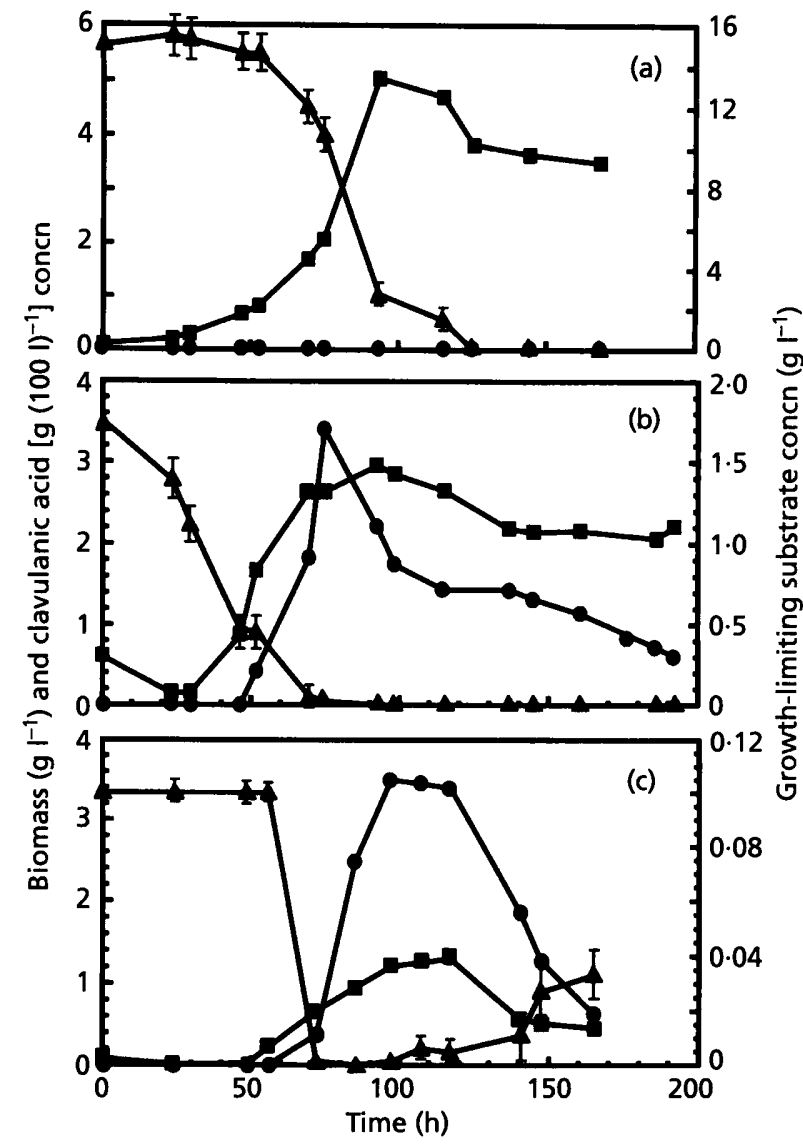

Fig. 1. Biomass concentration ( $\square$ ), clavulanic acid concentration (O) and growth rate-limiting substrate concentration $(\boldsymbol{A})$ in (a) C-limited (b) N-limited and (c) P-limited bioreactor batch culture.

obtained under $\mathrm{N}$ limitation $[0.01 \mathrm{~g}$ clavulanic acid $(\mathrm{g}$ biomass) $)^{-1}$.

\section{Effect of ammonium chloride concentration}

An optimum value for biomass yield at an $\mathrm{NH}_{4} \mathrm{Cl}$ concentration of $8 \mathrm{~g} \mathrm{l}^{-1}$ was observed in the P-limited medium over the range $4-20 \mathrm{~g} \mathrm{NH}_{4} \mathrm{Cl} \mathrm{l}^{-1}$. Varying the $\mathrm{KH}_{2} \mathrm{PO}_{4}$ concentration confirmed that phosphate was the growth-limiting substrate throughout this experiment. It was, therefore, assumed that ammonia was inhibitory to growth.

Reports of arginase activity in S. clavuligerus (Romero et al., 1986; Baldwin et al., 1994; Bascaran et al., 1989) are strongly suggestive of a full urea cycle (Mendz \& Hazell, 1997). A urea cycle in prokaryotes is unusual and is required for removal of ammonia from cells in which toxic intracellular concentrations are likely to occur, such as those of species that carry out rapid amino acid catabolism (Mendz \& Hazell, 1996). Sensitivity to ammonia concentration and presence of a urea cycle may, therefore, be linked. The urea cycle provides a very effective pathway for arginine biosynthesis so it is, perhaps, not surprising that a pathway for synthesizing an antibiotic using arginine as a precursor has evolved in this species.

\section{Effect of growth-rate down-regulation}

To study further the effect of ammonia, and provide a non-inhibitory culture system for complete ammonia utilization, a two-stage $\mathrm{NH}_{4} \mathrm{Cl}$-limited chemostat culture was operated. With a feed $\mathrm{NH}_{4} \mathrm{Cl}$ concentration of $3.8 \mathrm{~g} \mathrm{l}^{-1}, 2 \cdot 88 \mathrm{~g} \mathrm{l}^{-1}$ was utilized in stage 1 and a further $0.79 \mathrm{gl}^{-1}$ in stage 2 at a dilution rate of $0.05 \mathrm{~h}^{-1}$ (Table 1 ).

Assuming non-competitive substrate inhibition, it was possible to calculate values for substrate affinity constant $\left(K_{\mathrm{s}}\right)$ of $1.71 \mathrm{~g} \mathrm{l}^{-1}$ and inhibition constant $\left(K_{\mathrm{i}}\right)$ of $0.54 \mathrm{~g}$ $1^{-1}$. Thus, at $\mathrm{NH}_{4} \mathrm{Cl}$ values approaching these values, significant effects on, respectively, stimulation and inhibition of growth would be expected, consistent with our finding of an optimum ammonia concentration in batch culture of $8 \mathrm{~g} \mathrm{l}^{-1}$. Substituting an $\mathrm{NH}_{4} \mathrm{Cl}$ concentration of $8 \mathrm{~g} \mathrm{l}^{-1}$ into equation (2) indicates that, even at this optimum concentration, the growth rate is only 0.052 times $\mu_{\max }$.

The clavulanic acid concentration, and, therefore, production rate, increased by $161 \%$ from the first to the second stage. The production rate, relative to ammonia consumption, however, increased by $488 \%$ (Table 1 ). Had the relative production rate remained constant between stage 1 and stage 2 , the concentration of clavulanic acid in stage 2 would have increased by $1.93 \mathrm{mg} \mathrm{l}^{-1}$ to $5.03 \mathrm{mg} \mathrm{l}^{-1}$ (Table 1). The observed increase to $8 \cdot 1 \mathrm{mg} \mathrm{l}^{-1}$ suggests that the change in conditions between stage $1\left(\mu=0.05 \mathrm{~h}^{-1}\right)$ and stage $2(\mu$ $\left.=0.022 \mathrm{~h}^{-1}\right)$ stimulated the rate of antibiotic production. The specific ammonia uptake rate is decreased in stage 2. A possible explanation for the increased production is, therefore, that the clavulanic acid biosynthetic pathway is subject to ammonia repression and that this is relieved by the lower uptake rate in stage 2 . Inhibition of clavulanic acid biosynthesis by ammonia has been reported by Romero et al. (1984). This nonstoichiometric increase in antibiotic production is, however, also consistent with our findings in other fermentation systems in which growth rate is downregulated, such as the cyclic fed batch culture system (Bushell et al., 1997; Lynch \& Bushell, 1995).

The two-stage system was successful in utilizing the residual ammonia resulting from substrate limitation, and provided a disproportionate increase in the rate of clavulanic acid production arising as a consequence of down-regulating growth rate from $0.05 \mathrm{~h}^{-1}$ to $0.022 \mathrm{~h}^{-1}$ and/or decreasing the ammonia uptake rate between the first and second stages.

\section{Relationship between antibiotic production and amino acid pool size}

As the highest clavulanic acid production was obtained in P-limited batch culture, P-limited chemostat culture was used to investigate the relationship between in- 
Table 1. Data from two-stage $\mathrm{N}$-limited chemostat culture

The theoretical increase in clavulanic acid concentration in stage 2 is $1.93 \mathrm{mg} \mathrm{l}^{-1}\left\{\left[\mathrm{mg}\left(\mathrm{g} \mathrm{NH}_{4}^{+}\right)^{-1} \mathrm{~h}^{-1}\right.\right.$ in stage $1 \times \mathrm{NH}_{4}^{+}$used in stage 2]/(growth rate in stage 2)\}; the theoretical clavulanic acid concentration in stage 2 is, therefore, $5.03 \mathrm{mg} \mathrm{l}^{-1}$ (theoretical concentration in stage 2 plus observed concentration in stage 1 ).

\begin{tabular}{|c|c|c|c|}
\hline & Stage 1 & Stage 2 & Notes \\
\hline Dilution rate $\left(\mathrm{h}^{-1}\right)$ & $0 \cdot 050$ & $0 \cdot 050$ & Observation \\
\hline Specific growth rate $\left(\mathrm{h}^{-1}\right)$ & $0 \cdot 050$ & 0.022 & From equation $(1)$ \\
\hline Residual $\mathrm{NH}_{4} \mathrm{Cl}$ concn $\left(\mathrm{g} \mathrm{l}^{-1}\right)$ & $0 \cdot 92$ & $0 \cdot 13$ & Observation \\
\hline $\mathrm{NH}_{4} \mathrm{Cl}$ used $\left(\mathrm{g} \mathrm{l}^{-1}\right)$ & $2 \cdot 88$ & 0.79 & Inflow minus residual \\
\hline $\begin{array}{l}\text { Clavulanic acid concn } \\
\mathrm{mg} \mathrm{l}^{-1} \\
\% \text { increase }\end{array}$ & $3 \cdot 1$ & $\begin{array}{l}8 \cdot 1 \\
161\end{array}$ & $\begin{array}{l}\text { Observation } \\
100 \times(\text { stage } 2-\text { stage } 1) / \text { stage } 1\end{array}$ \\
\hline $\begin{array}{l}\text { Clavulanic acid production rat } \\
\mathrm{mg} \mathrm{l}^{-1} \mathrm{~h}^{-1} \\
\mathrm{mg}\left(\mathrm{g} \mathrm{NH}_{4}^{+}\right)^{-1} \mathrm{~h}^{-1} \\
\% \text { increase }\end{array}$ & $\begin{array}{l}0.155 \\
0.054\end{array}$ & $\begin{array}{c}0.405 \\
0.316 \\
485\end{array}$ & $\begin{array}{l}\text { Concn } \times \text { dilution rate } \\
(\text { Concn } \times \text { dilution rate }) / \mathrm{NH}_{4}^{+} \text {used } \\
100 \times(\text { stage } 2-\text { stage } 1) / \text { stage } 1\end{array}$ \\
\hline
\end{tabular}

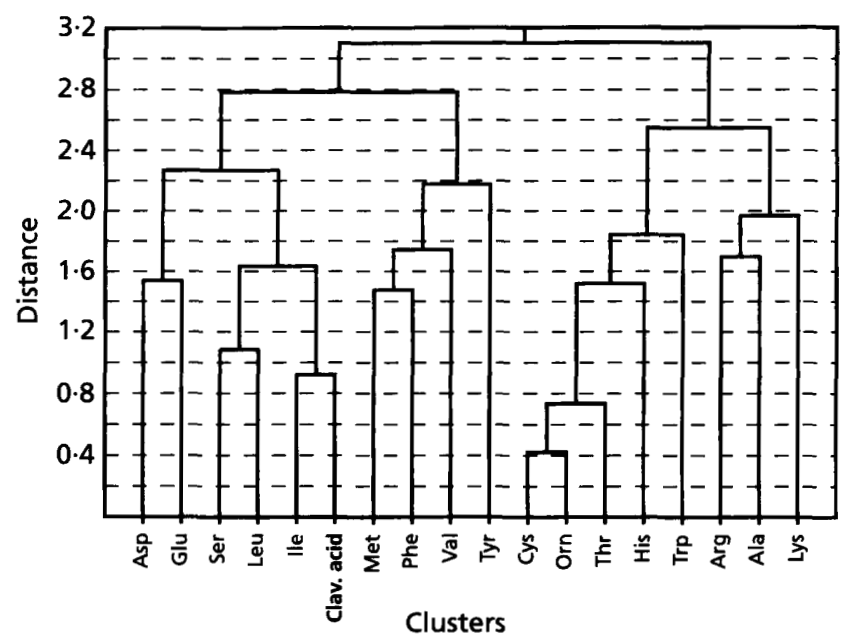

Fig. 2. Single-linkage dendrogram obtained by analysing standardized intracellular amino acid and clavulanic acid production rates over a range of dilution rates between $0.03 \mathrm{~h}^{-1}$ and $0.09 \mathrm{~h}^{-1}$ (see Methods). tracellular amino acid production rate and clavulanic acid production.

The intracellular amino acid content was quantified over the growth rate range $0.03-0.09 \mathrm{~h}^{-1}$ and the correlation between values obtained and clavulanic acid production was quantified using cluster analysis (Fig. 2). To test the physiological validity of this analysis, the effect of supplementing the medium with arginine, isoleucine, leucine, serine or valine (to give a final concentration of $10 \mathrm{mM}$ ) was examined in chemostat cultures growing at a dilution rate of $0.03 \mathrm{~h}^{-1}$. Valine, serine, leucine and isoleucine feeds resulted in increased clavulanic acid production compared to the control (Table 2).

\section{Hypotheses to account for the effect of amino acid feeds on antibiotic production}

If it is assumed that the supply of $C_{3}$ precursor is rate limiting for clavulanic acid production whereas the supply of $\mathrm{C}_{5}$ precursor is not, it can be postulated that

Table 2. Effect of amino acid feeds on the specific productivity of clavulanic acid in a P-limited chemostat culture $\left(D=0.03 \mathrm{~h}^{-1}\right)$

\begin{tabular}{|lcc|}
\hline $\begin{array}{l}\text { Amino acid in feed } \\
\text { (final concn } \mathbf{1 0} \mathbf{~ m M})\end{array}$ & \multicolumn{2}{c|}{ Clavulanic acid titre } \\
\cline { 2 - 3 } & {$\left[\mathbf{m g}(\mathbf{m g} \text { biomass })^{-\mathbf{1}}\right]$} & \% of control \\
\hline Control (none) & 0.022 & 100 \\
Arginine & 0.022 & 177 \\
Isoleucine & 0.039 & 177 \\
Leucine & 0.039 & 177 \\
Serine & 0.039 & 145 \\
Valine & 0.032 & \\
\hline
\end{tabular}




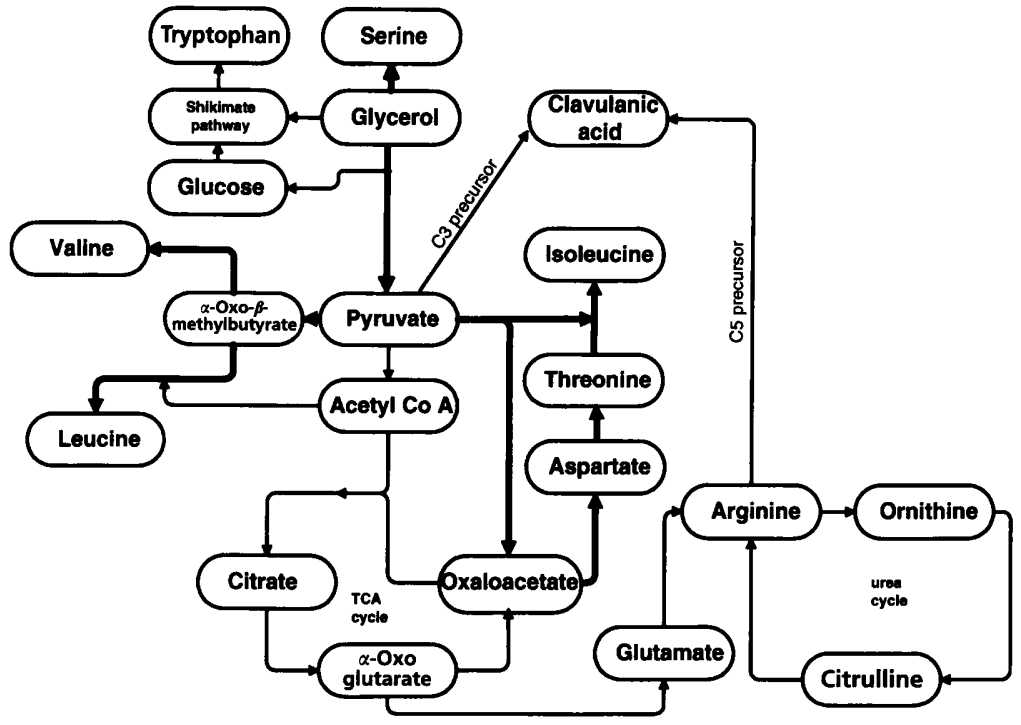

Fig. 3. Postulated biosynthetic scheme for clavulanic acid production, illustrating major carbon flux (many intermediate steps omitted). Heavy arrows indicate the fluxes competing with clavulanic acid production that were influenced by the amino acid feeds. feeding leucine or isoleucine will decrease the anaplerotic carbon flux of pyruvate to the synthesis of these amino acids, thus allowing more $\mathrm{C}_{3}$ precursor for the synthesis of clavulanic acid (Fig. 3). Increased leucine and isoleucine pool sizes were observed in the fed cultures (Fig. 4d, e) and the synthesis of both amino acids is repressed in many microbial species under these circumstances (Umbarger, 1978). The increase in the ornithine pool size in both cases is consistent with the assumption that the pool size of arginine (its immediate precursor) is not rate limiting and the decrease in arginine pool size is consistent with an increased rate of clavulanic acid production (Fig. 4d, e).

The valine and serine feeds, which resulted in an increase in intracellular valine and serine concentration, respectively (Fig. 4b, c), would also have allowed an increase in the supply of $\mathrm{C}_{3}$ precursor for clavulanic acid synthesis (Fig. 3). Presumably the valine feed was not as effective as the leucine feed in enhancing antibiotic production because leucine requires carbon skeleton from both $\alpha$-oxo- $\beta$-methylbutyrate (derived from pyruvate) and acetyl-CoA (also derived from pyruvate) and, therefore, accounts for a greater drain on $\mathrm{C}_{3}$ precursor. It should be noted that the synthesis of the pyruvate family (leucine, isoleucine and valine) can be repressed by any of that group (Umbarger, 1978).

Further evidence that $\mathrm{C}_{3}$ carbon supply is rate-limiting rather than $\mathrm{C}_{5}$ was obtained with the arginine feed, which resulted in an increase in the pool size of arginine (Fig. 4a), the immediate precursor of clavulanic acid (Valentine et al., 1993), without increasing clavulanic acid production. Limitation of clavulanic acid synthesis due to $C_{3}$ supply is also consistent with the effect of growth-rate-limiting nutrient on antibiotic production (Fig. 1). Under P limitation, which supports the highest clavulanic acid production, gluconeogenesis and glycolysis would be restricted by the supply of phosphate, restricting the flux of glycerol into these pathways and allowing the flux of $\mathrm{C}_{3}$ precursor into clavulanic acid to increase. Under $\mathrm{N}$ limitation, these restrictions would not apply, so clavulanic acid production would have to compete with gluconeogenesis and glycolysis. Under C limitation, the resultant restriction in glycerol assimilation would limit the global supply of $\mathrm{C}_{3}$, presumably accounting for the abolition of clavulanic acid production in a biosynthesis where $C_{3}$ supply is rate limiting.

Feeds of arginine and valine resulted in an increase in glutamate pool size. This may be a reflection of the decreased anaplerotic flux, required for their syntheses, which presumably has the effect of decreasing $C_{3}$ flux into pyruvate, discussed above. All feeds also resulted in an increase in intracellular tryptophan and tyrosine concentrations, indicating the activity of the shikimate pathway in this organism (Fig. 3). As the shikimate pathway requires both $\mathrm{C}_{3}$ and $\mathrm{C}_{6}$ precursors (the latter having to be produced by gluconeogenesis since $S$. clavuligerus has impaired glucose-utilizing ability) this would provide considerable competition for $\mathrm{C}_{3}$ precursor.

We have not quantified the production of other antibiotics. It is possible that the feeding experiments could have influenced the production of these compounds, particularly where amino acids in the feed are acting as biosynthetic precursors.

\section{Concluding remarks}

Clavulanic acid was produced as a secondary metabolite following exponential phase in batch culture and in response to growth-rate down-regulation in two-stage chemostat culture. This latter technique allowed efficient usage of ammonia, despite the fact that it appears to be a growth-inhibitory substrate.

Cluster analysis may be used to identify those intermediary metabolites that are produced at rates most 


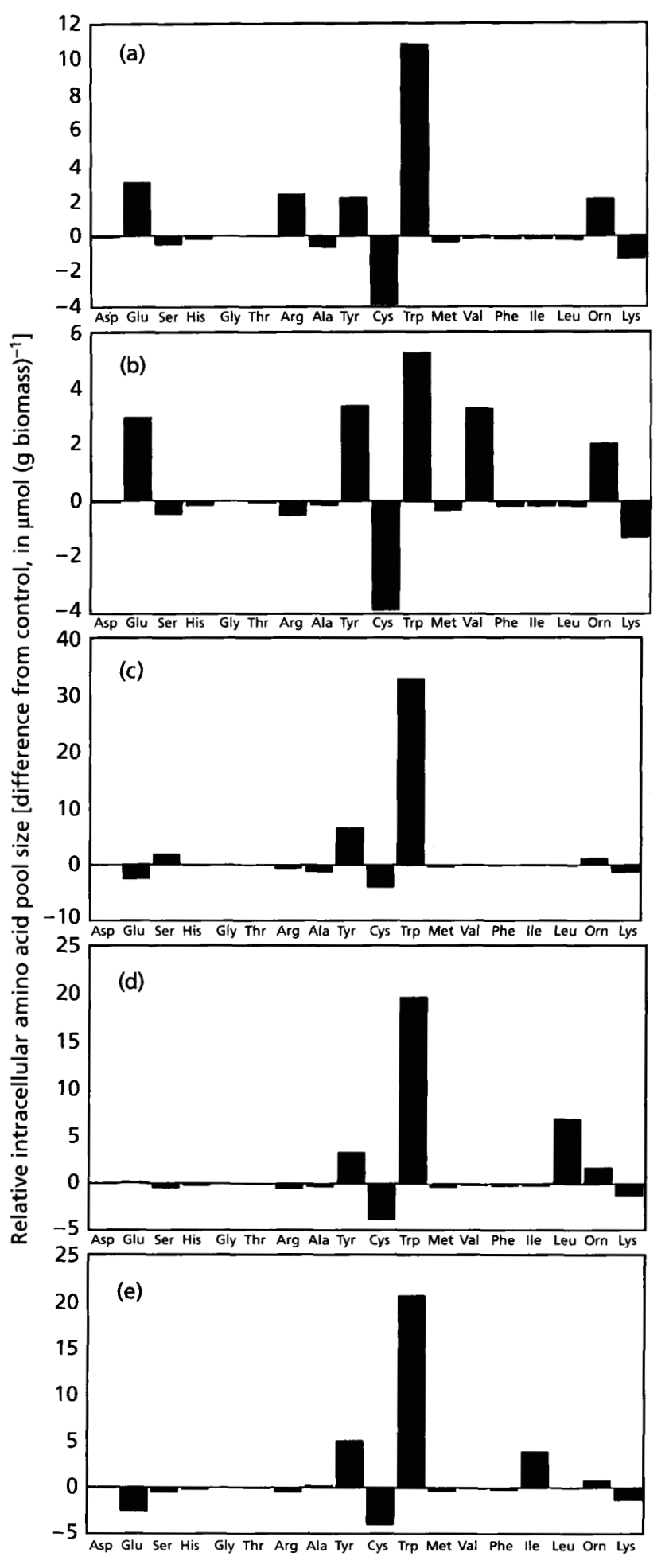

Fig. 4. Intracellular amino acid pool size, shown as $\mu \mathrm{mol}$ (g biomass) ${ }^{-1}$ above or below the control value (no extra feed; 0 on $y$ axis), of a chemostat culture at a dilution rate of $0.03 \mathrm{~h}^{-1}$. Supplemented feed solutions contained $10 \mathrm{mM}$ (final concentration) of: (a) arginine, (b) valine, (c) serine, (d) leucine, (e) isoleucine. closely correlated with antibiotic biosynthesis. This information has been used to construct culture feeds resulting in enhanced antibiotic production. This approach may have potential as a generic method for formulating feeds that are capable of enhancing product formation by influencing biosynthetic pathway fluxes. An advantage of this approach is that no knowledge of the biosynthetic pathway would be required to construct the feed.

The effect of various feeds on intracellular amino acid pool sizes indicated that manipulation of physiology to enhance clavulanic acid production requires alleviation of competition from other pathways for a $\mathrm{C}_{3}$ precursor, the availability of which is critical due to the organism's impaired ability to assimilate glucose. The unusual presence of a urea cycle in a prokaryote would provide an abundant supply of arginine, the $C_{5}$ precursor to clavulanic acid.

\section{REFERENCES}

Baldwin, J. E., Goh, K.-C. \& Schofield, C. J. (1994). Biosynthetic precursors of valclavam. Tetrahedron Lett 35, 2779-2782.

Bascaran, V., Hardisson, C. \& Braña, A. F. (1989). Isolation and characterization of nitrogen-deregulated mutants of Streptomyces clavuligerus. J Gen Microbiol 135, 2475-2482.

Brown, A. G. (1986). Clavulanic acid, a novel $\beta$-lactamase inhibitor: a case study in drug discovery and development. Drug Design Deliv 1, 1-21.

Bushell, M. E. (1988). Growth, metabolism and fermentation technology. In Biotechnology of the Actinomycetales, pp. 185-215. Edited by M. Goodfellow, S. T. Williams \& M. Mordarski. London: Academic Press.

Bushell, M. E., Bell, S. L., Scott, M. F., Spier, R. E., Wardell, J. N. \& Sanders, P. G. (1994). Enhancement of monoclonal antibody yield by hybridoma fed-batch culture, resulting in extended maintenance of viable cell population. Biotechnol Bioeng 44, 1099-1106.

Bushell, M. E., Smith, J. \& Lynch, H. C. (1997). A physiological model for the control of erythromycin production in batch and cyclic fed batch culture. Microbiology 143, 475-480.

Clark, G. J., Langley, D. \& Bushell, M. E. (1995). Oxygen limitation can induce microbial secondary metabolite formation: investigations with miniature electrodes in shaker and bioreactor culture. Microbiology 141, 663-669.

Foulstone, M. \& Reading, C. (1982). Assay of Amoxicillin and clavulanic acid, the components of Augmentin, in biological fluids with high performance liquid chromatography. Antimicrob Agents Chemother 22, 753-762.

Hopwood, D. A. (1981). Genetic studies of antibiotics and other secondary metabolites. In Genetics as a Tool in Microbiology (Symposia of the Society for General Microbiology vol. 31), pp. 187-218. Edited by S. W. Glover \& D. A. Hopwood. Cambridge: Cambridge University Press.

Ives, P. R. (1995). Physiological aspects of the production of clavulanic acid by Streptomyces clavuligerus. PhD thesis, University of Surrey.

Jones, B. N., Paabo, S. \& Stein, S. (1981). Amino acid analysis and enzymatic sequence determination of peptides by an improved $o$ phthaldiamide precolumn labelling procedure. J Liq Chromatogr 4, 565-586. 
Joseph, M. H. \& Marsden, C. A. (1986). Amino acids and small peptides. In HPLC of Small Molecules : a Practical Approach, pp. 13-28. Edited by C. K. Lim. Oxford: IRL Press.

Lynch, H. C. \& Bushell, M. E. (1995). The physiology of erythromycin biosynthesis in cyclic fed batch culture. Microbiology 141, 3105-3111.

McDermott, J. F., Lethbridge, G. \& Bushell, M. E. (1993). Estimation of the kinetic constants and elucidation of trends in growth and erythromycin production in batch and continuous cultures of Saccharopolyspora erythraea using curve fitting techniques. Enzyme Microb Technol 15, 657-663.

Mendz, G. L. \& Hazell, S. L. (1996). The urea cycle of Helicobacter pylori. Microbiology 142, 2959-2967.

Pirt, S. J. (1975). Principles of Microbe and Cell Cultivation. Oxford: Blackwell Scientific Publications.

Pitlik, J. \& Townsend, C. A. (1997). The fate of [2,3,3-H-2(3), 1,2C-13(2)]-D,L-glycerate in clavulanic acid. J Chem Soc Chem Commun 2, 225-226.

Queener, S. \& Swartz, R. (1979). Penicillins; biosynthetic and semisynthetic. In Economic Microbiology, vol. 3, Secondary Products of Metabolism, pp. 35-123. Edited by A. H. Rose. London: Academic Press.

Reading, C. \& Cole, M. (1977). Clavulanic acid: a $\beta$-lactamase inhibiting $\beta$-lactam from Streptomyces clavuligerus. Antimicrob Agents Chemother 11, 852-857.

Reed, G. \& Peppler, H. J. (1973). Yeast Technology, pp. 664-668. Westport: AVI.
Romero, J., Liras, P. \& Martin, J. F. (1984). Dissociation of cephamycin and clavulanic acid production in Streptomyces clavuligerus. Appl Microbiol Biotechnol 20, 318-325.

Romero, J., Liras, P. \& Martin, J. F. (1986). Utilisation of ornithine and arginine as specific precursors of clavulanic acid. Appl Environ Microbiol 52, 892-897.

Stirling, I. \& Elson, S. W. (1979). Studies on the biosynthesis of clavulanic acid. II. Chemical degradations of ${ }^{14} \mathrm{C}$-labelled clavulanic acid. J Antibiot 32, 1125-1129.

Townsend, C. A. \& Ho, M.-F. (1985a). Biosynthesis of clavulanic acid: origin of the $C_{5}$ unit. J Am Chem Soc 107, 1065-1066.

Townsend, C. A. \& Ho, M.-F. (1985b). Biosynthesis of clavulanic acid: origin of the $\mathrm{C}_{3}$ unit. J Am Chem Soc 107, 1066-1068.

Umbarger, H. E. (1978). Amino acid biosynthesis and its regulation. Annu Rev Biochem 47, 533-606.

Valentine, B. P., Bailey, C. R., Doherty, A., Morris, J., Elson, S. W., Baggaley, K. H. \& Nicholson, N. H. (1993). Evidence that arginine is a later metabolic intermediate than ornithine in the biosynthesis of clavulanic acid by Streptomyces clavuligerus. J Chem Soc Chem Commun 15, 1210-1211.

Wilson, G. C. \& Bushell, M. E. (1995). The induction of antibiotic synthesis in Saccharopolyspora erythraea and Streptomyces hygroscopicus by growth rate decrease is accompanied by a down-regulation of protein synthesis rate. FEMS Microbiol Lett 129, 89-96.

Received 13 May 1997; revised 30 June 1997; accepted 30 July 1997. 